\title{
Hersteller erhält Gründerpreis
}

Die GME German Medical Engineering $\mathrm{GmbH}$ hat sich mit kleinen, leistungsstarken Lasern für dermatologische und ästhetische Anwendungen weltweit etabliert und dafür den mit 10.000 Euro dotierten Gründerpreis der Industrieund Handelskammer Mittelfranken erhalten.

95\% der Systeme gehen in den Export. Wichtigster Absatz sind die USA. Der Haarentfernungslaser LinScan 808 wird dort unter dem Namen „Clear + Brilliant“ Pélo von Solta Medical vertrieben.

Mit Lasern kennt sich GME bestens aus: Der Volkswirt Stefan Schulze und der Physiker Dietmar Fischer waren viele Jahre gemeinsam beim Laserhersteller WaveLight (später Quantel) beschäftigt, als sie 2011 zusammen mit dem Arzt und Physiker Dieter Manstein als klinischen Berater ihr Unternehmen gründeten und die Entwicklungsmannschaft mitnahmen.
Inzwischen beschäftigt GME zwölf Mitarbeiter und hat im vergangenen Jahr bereits die Gewinnzone erreicht. Etwa 170 der rund 20.000 Euro teuren Geräte hat das Unternehmen 2015 verkauft, im laufenden Jahr sollen es sogar $50 \%$ mehr sein. Drei verschiedene Lasertypen hat GME heute im Angebot: Einen fraktionalen $\mathrm{CO}_{2}$-Laser, einen Dioden-Laser für die Epilation und ein EPL Excimer Pulsed Light System zur Behandlung von Psoriasis und Vitiligo. Mehr Informationen unter www.gmeonline.de

red

Nach Informationen von GME German Medical Engineering

\section{Zusammenhang zwischen Bakterien und Neurodermitis}

Eine im British Journal of Dermatology veröffentlichte Studie [Totté JE et al. Br J Dermatol 2016;175:687-5] hat gezeigt, dass durchschnittlich $70 \%$ der Neurodermitispatienten mit Staphylococcus-aureus-Bakterien (S. aureus, einschließlich MRSA) in ihren Hautläsionen besiedelt sind. Diese Ergebnisse weisen auf die Bedeutung der Besiedlung als einer der möglichen Auslöser für atopische Dermatitis hin.

In einer systematischen Literaturstudie an der Erasmus Universitätsklinik, Rotterdam, wurden 95 Beobachtungsstu- dien mit über 9.000 Patienten ausgewertet. S. aureus fand sich bei $39 \%$ der Neurodermitispatienten auf gesunder Haut und $\mathrm{zu} 70 \%$ im Bereich ihrer Hautläsionen wieder - eine fast 20-fache Steigerung im Vergleich zu gesunden Kontrollen.

Zusätzlich hatten bis zu $80 \%$ der Neurodermitispatienten einen S.-aureusStamm, der einen Giftstoff produzierte. Diese Giftstoffe sind dafür bekannt, Entzündungsreaktionen auszulösen. Bei Neurodermitis tragen sie zu den Hautbarriereschädigungen bei und können daher primäre Auslöser der Erkrankung sein.
Die Firma Micreos, die die Literaturstudie unterstützt hat, ist wegweisend mit der Entwicklung von Staphefekt ${ }^{\mathrm{Tm}}$, einem bakterienabtötenden Enzym (Endolysin), das spezifisch gegen $S$. aureus wirkt. Es tötet MRSA ebenso wie andere S.-aureusStämme effektiv ab.

Eine bakterielle Resistenz gegenüber Endolysin wurde, im Gegensatz zu Antibiotika, nicht festgestellt. Nützliche Bakterien bleiben erhalten, sodass es sich für die tägliche Langzeitanwendung eignet.

Das Mittel ist das erste Endolysin, das für die Anwendung auf dem Mikrobiom der Haut registriert ist und derzeit als aktiver Wirkstoff in der Produktlinie Gladskin (topische Salben und Gele für entzündliche Hauterkrankungen wie auch Neurodermitis) eingesetzt wird. red

Nach Informationen von Micreos

\section{Creme-Foundation mit hoher Deckkraft}

Bio-Fond ist eine hypoallergene CremeFoundation mit hoher Deckkraft. Die Inhaltsstoffe Lecithin und Tocopherol fördern den Hautstoffwechsel und unterstützen die Bindegewebsfestigkeit und Zellneubildung. Olivensqualan bewahrt die Haut vor Feuchtigkeitsverlust, verhindert die Oxidation hauteigener Fette und sorgt für ein angenehmes Hautgefühl. Weitere hochwertige Inhaltsstoffe wie z. B. Vitamin E runden das pflegende Make-up dermatologisch anspruchsvoll ab.
Schon eine geringe Menge garantiert eine vollständige Abdeckung von Rosacea und Couperose. Auch Alters- und Pigmentflecken oder Narben werden unsichtbar. Zudem ist das Produkt dank seiner umfassenden Farbpalette für fast alle Teints geeignet. Alle Produkte von Gerda Spillmann sind parabenfrei, enthalten keine Silikone und kommen ohne Tierversuche aus. Die Erzeugnisse der Schweizer Firma sind frei von Konservierungsstoffen und Nanopartikeln. Alle
Roh-, Nähr- und Wirkstoffe sind fast ausschließlich pflanzlichen Ursprungs und ihre positive Wirkung ist wissenschaftlich belegt.

Worauf Hollywoods Make-up-Artists seit Jahren schwören und was führende Dermatologen empfehlen, ist nun auf dem deutschen Markt in ausgewählten Apotheken und im Online-Shop unter www.gerda-spillmann.de erhältlich.

Der geschäftsführende Gesellschafter Thomas Schachler freut sich über Fragen oder Anregungen (E-Mail: t.schachler@ gerdaspillmann.de).

Nach Informationen von Gerda Spillmann Deutschland 